\title{
Escalas de dor no paciente crítico: uma revisão integrativa
}

\author{
Scales of pain in the critically ill patient: \\ an integrative review
}

\author{
Juliana G. S. Fortunato* \\ Monique de S. Furtado \\ Leni F. de Assis Hirabae \\ Josiana A. de Oliveira
}

\section{Resumo}

A dor deve ter grande importância para a enfermagem nos cenários hospitalares, pois é considerada o quinto sinal vital. Os pacientes manifestam dor de diversas maneiras e existem escalas que a avaliam a fim de conduzir a terapêutica adequada. Pacientes críticos merecem destaque, pois devido ao quadro clínico e ao ambiente que estão submetidos podem ter dificuldades de expressar fisicamente dor. A maioria desses clientes experimenta dor, medo e ansiedade e isto pode retardar, por exemplo, a recuperação e a liberação da ventilação mecânica. Desta forma, o alívio da dor mostra-se essencial para auxiliar num melhor prognóstico. Os objetivos do estudo são realizar revisão bibliográfica de produções científicas existentes sobre as escalas de dor e discutir o uso das escalas de dor encontradas na revisão bibliográfica, no período de 2008 a 2012, em pacientes de terapia intensiva. Este estudo é uma revisão bibliográfica integrativa, com busca em duas bases de dados on-line, em novembro de 2012, obedecendo aos seguintes critérios: estar em português, enquadrar-se entre 2008 e 2012, ter compatibilidade com o tema, estar na íntegra e disponível gratuitamente. Foram excluídas aquelas produções que não obedeceram a estes critérios e que apareceram repetidamente. A seleção resultou em 12 publicações científicas. Foram encontradas e discutidas cinco tipos de escalas: visual/verbal numérica, visual analógica, faces de dor, dor comportamental e questionário de dor McGill. Portanto, a equipe de enfermagem precisa conhecer e entender as escalas disponíveis para saber escolher a melhor, adequada ao seu tipo de paciente.

Descritores: Terapia intensiva; Dor; Enfermagem. 


\section{Abstract}

The pain must have great importance for nursing in hospital settings because it is considered the fifth vital sign. Customers express grief in different ways and there are scales that assess to conduct appropriate therapy. Critically ill patients are noteworthy because, due to the clinical environment to wich it is submitted, may struggle to express pain physically. Most of these customers experience pain, fear and anxiety, and this can slow down, for example, recovery and liberation from mechanical ventilation. Thus, pain relief proves essential to aid a better prognosis. The study objectives are: to review the literature in the period from 2008 to 2012, productions of existing science on pain scales and discuss the use of pain scales found in the literature review from 2008 to 2012 in patients intensive care. This study is an integrative literature review, with two search databases online, in November 2012, following the criteria: to be in Portuguese, to fit between 2008 and 2012, to have compatibility with the theme and to be in full available for free. We excluded those productions that did not obey these criteria and that appeared repeatedly. The selection resulted in twelve scientific publications. There were found and discussed five types of scales: Visual /Verbal Numerical, Visual Analogue, Faces of Pain, Pain and Behavioral McGill Pain Questionnaire. Therefore, the nursing staff must know and understand the scales available to know how to choose the best to suit your type of patient.

Keywords: Intensive care; Pain; Nursing.

\section{Introdução}

De acordo com a International Association for the Study of Pain (IASP) a dor é definida como uma experiência sensorial e emocional desagradável associada a um dano atual ou potencial do tecido. Desta forma, em 1996, foi introduzida pela American Pain Society, como $5^{\circ}$ sinal vital. ${ }^{1}$

A dor é algo comum a todo ser humano, que possui suas particularidades e pontos-chave, sendo por vezes ativada por componentes psicológicos e subjetivos. Assim sendo, cada indivíduo reage a dor de forma diferente, levando também em consideração a intensidade da dor.

Tendo em vista que a dor é algo subjetivo e que cada pessoa se expressa de forma diferente, o primeiro desafio no combate à dor é sua mensuração. ${ }^{1}$ Neste intuito foram criadas diversas escalas a fim de mensurar a dor do indivíduo para que uma intervenção seja feita. No entanto, é preciso saber escolher a escala que melhor se enquadra ao paciente em questão.

O processo de avaliação da dor é amplo e envolve a obtenção de informações relacionadas à data de início, à localização, à intensidade, à duração e à periodicidade dos episódios dolorosos, às qualidades sensoriais e afetivas do paciente, aos fatores que iniciam, aumentam ou diminuem a sua intensidade. Sendo assim, o alívio da dor é um pré-requisito para que o paciente obtenha uma ótima recuperação e qualidade de vida. ${ }^{2}$

O indivíduo hospitalizado possui diversos fatores que podem influenciar seu estado psicológico e com isso alterar ou intensificar sua representação de dor, como o medo de se submeter a certos procedimentos, medo da morte e de estar longe da família, por exemplo. No entanto, quando este consegue se comunicar fica mais fácil para o profissional identificar o que o está afetando e tentar sanar o problema. Porém, quando o paciente não está em condições de falar ou sinalizar o problema há um grande entrave para a identificação deste.

Os pacientes internados em unidades de terapia intensiva (UTIs) são exemplos de pacientes que dificilmente conseguem expressar sua dor. Isso se deve à gama de sedativos que recebem por conta de sua condição clínica. A maioria deste tipo de paciente experimenta dor, medo e ansiedade, o que pode retardar a recuperação e a liberação da ventilação mecânica. ${ }^{3}$ Desta forma, o alívio da dor mostra-se essencial para a recuperação adequada.

Com o intuito de discutir as formas de avaliação da dor, através de escalas, dos pacien- 
tes hospitalizados em UTIs, o presente estudo objetiva:

- realizar revisão bibliográfica, no período de 2008 a 2012, de produções científicas existentes sobre as escalas de dor;

- discutir o uso das escalas de dor encontradas na revisão bibliográfica no período de 2008 a 2012 em pacientes de terapia intensiva.

Tal proposta se faz justificável, pois o profissional de enfermagem pode apresentar dificuldades em lidar com as queixas álgicas dos pacientes. ${ }^{2}$

Este estudo é relevante para a produção científica da enfermagem, já que a defasagem de conhecimento nesta área propicia um inadequado reconhecimento e tratamento da dor. ${ }^{4}$

\section{Procedimentos metodológicos}

Este estudo é uma revisão bibliográfica do tipo integrativa, que é definida por um método de revisão mais amplo, permitindo inclusão de literatura teórica e empírica, bem como estudos com diferentes abordagens metodológicas (quantitativa e qualitativa). Os estudos inclusos são analisados de forma sistemática, ou seja, através de um protocolo pré-estabelecido, que deve orientar todo o processo. ${ }^{5}$
Uma revisão integrativa deve obedecer ao seguinte processo: escolha do tema, busca na literatura (amostragem), critérios para a categorização do estudo (coleta de dados), avaliação dos estudos incluídos nos resultados, discussão do resultado e apresentação da revisão integrativa. ${ }^{6}$

Desta maneira, foi realizada revisão bibliográfica em duas bases de dados on-line (SciELO e LILACS), em novembro de 2012, obedecendo aos seguintes critérios: estar em português, enquadrar-se entre 2008 e 2012, ser compatível com o tema, estar na íntegra e disponível gratuitamente. Foram excluídos aqueles que não obedeceram a estes critérios e os que apareceram repetidamente em ambas as bases. As palavras-chave de acordo com o DeCS (descritores em ciências da saúde) foram: dor, terapia intensiva e enfermagem. A busca foi realizada usando: "dor and terapia intensiva" e "dor and enfermagem".

Após seguir os critérios supracitados a busca resultou na escolha de 12 publicações (Figura 1), para serem analisadas, discutidas e apresentadas posteriormente. Espera-se com essa análise fornecer maiores subsídios para atuação do enfermeiro junto ao paciente crítico e despertar o interesse do mesmo pelo estudo da identificação e controle da dor dos pacientes sob sua responsabilidade.

\section{SCIELO}

\begin{tabular}{|c|c|c|c|c|c|c|c|c|}
\cline { 2 - 8 } & Descritores & Português & $\begin{array}{c}\text { Ano de } \\
\text { publicaçấ }\end{array}$ & $\begin{array}{c}\text { Adequados à } \\
\text { temática }\end{array}$ & $\begin{array}{c}\text { Disponiveis } \\
\text { na } \\
\text { integgra }\end{array}$ & $\begin{array}{c}\text { Disponiveis } \\
\text { gratuitamente }\end{array}$ & Requetidos & Selecionados \\
\hline $\begin{array}{c}\text { Dor and } \\
\text { Terapia } \\
\text { Intersiva }\end{array}$ & 45 & 44 & 28 & 4 & 4 & 4 & 0 & 4 \\
\hline $\begin{array}{c}\text { Dor and } \\
\text { effemagem }\end{array}$ & 126 & 98 & 54 & 4 & 3 & 3 & 1 & 2 \\
\hline
\end{tabular}

LILACS

\begin{tabular}{|c|c|c|c|c|c|c|c|c|}
\hline $\begin{array}{c}\text { Dor and } \\
\text { Taraia } \\
\text { Intensiva }\end{array}$ & 160 & 118 & 62 & 4 & 4 & 4 & 1 & 3 \\
\hline $\begin{array}{c}\text { Dor and } \\
\text { enfemagem }\end{array}$ & 620 & 479 & 192 & 10 & 8 & 8 & 5 & 3 \\
\hline
\end{tabular}

Total de publicações selecionadas $=12$

Figura 1. Seleção da bibliografia para o estudo 


\section{Resultados e discussão}

Existem alguns fatores que comumente causam dor ao paciente em terapia intensiva, sendo eles: aspiração traqueal, mudança de decúbito, curativo, tubo traqueal, trauma, lesão cirúrgica, queimadura, sonda nasogástrica, cateter central, cateter arterial, drenos, escaras, punção venosa ou arterial e faixa para restrição dos membros, entre outros. $^{3}$

Quando a dor não é controlada podem ocorrer alterações respiratórias, hemodinâmicas e metabólicas, aumentando a probabilidade de ocorrer instabilidade cardiovascular, aumento do gasto energético e dificuldade de deambulação favorecendo a trombose venosa profunda. ${ }^{7}$

As escalas devem dar subsídios ao profissional para que ele identifique as alterações presentes no paciente e use a intervenção adequada. Isso implica no fato de que o profissional deve estar bem treinado a fim de saber aplicar a escala e interpretá-la. Pois, muito mais do que apontar a alteração presente, este tipo de instrumento deve nortear as ações, objetivando sempre a melhora da condição do paciente.

Com o intuito de discutir as escalas encontradas à luz do cuidado com paciente crítico serão apontadas as escalas indicadas nas publicações selecionadas para o presente estudo.

A primeira delas é a escala visual/verbal numérica (EVN) que objetiva a mensuração da intensidade da dor, em contextos clínicos, em valores numéricos. O paciente deve estar consciente de seus pensamentos e ações e referir sua dor numa escala de zero a dez, sendo zero "nenhuma dor" e dez a "dor máxima imaginável" (Figura 2). Possui uma expressão de dor precisa e é de fácil utilização. ${ }^{8}$

Esta escala não exige, obrigatoriamente, que haja contato visual do paciente com a escala, ou seja, ela pode ser simplesmente falada ao paciente, tornando-a simples para indivíduos analfabetos ou com dificuldade visual. Ressalta-se que é aplicável a pacientes orientados e com boa capacidade cognitiva, que num contexto de terapia intensiva, podem estar aguardando, por exemplo, um procedimento cirúrgico.
Outra escala citada é a escala visual analógica (EVA), que se assemelha à EVN; no entanto, deve obrigatoriamente haver o contato visual do paciente com a escala e ele deve ser capaz de apontar ou sinalizar ao examinador em que grau sua dor está. ${ }^{8}$ Pode ser uma régua numérica com dez centímetros, dividida em dez espaços iguais, sendo apresentada de forma simples, ou pode possuir um apelo visual com cores, mas é importante que o paciente entenda que uma extremidade indica "sem dor" e que a outra indica "dor máxima" (Figura 3).

Assim como a EVN, esta exige que o paciente esteja orientado, com boa acuidade visual e boa capacidade cognitiva, o que a torna de difícil aplicação em cenários de terapia intensiva, principalmente em idosos.

A prevalência de idosos na UTI e a intensidade de sua dor é muito grande e está associada a desordens crônicas, como doenças musculoesqueléticas (artrite e osteoporose). Além disso, também estão mais expostos à alta incidência de câncer, necessidade de procedimentos cirúrgicos, úlceras por pressão e as doenças cardiovasculares. ${ }^{9}$

A escala de faces de dor (EFD) se vale de descritores visuais através de expressões faciais que refletem a intensidade da dor. O indivíduo é convidado a analisar as imagens e indicar qual delas se relaciona à dor que o mesmo está sentindo. ${ }^{8}$ Varia de zero a cinco, sendo zero "sem dor" e cinco "dor insuportável" (Figura 4).

Baseado nisto, infere-se que esta escala é limitada em um cenário de terapia intensiva pelo fato de que estes pacientes geralmente estão submetidos a uma grande quantidade de sedativos. Estes podem interferir na capacidade do indivíduo em responder fisicamente a estímulos dolorosos, ou seja, mesmo que esteja com algum desconforto, poderá ter dificuldades de expressar faces de dor. No entanto, é possível que esta dor se manifeste de outras maneiras; por exemplo, na elevação da pressão arterial, elemento que não é contemplado na utilização das escalas mencionadas.

Mesmo que o profissional pudesse realizar 


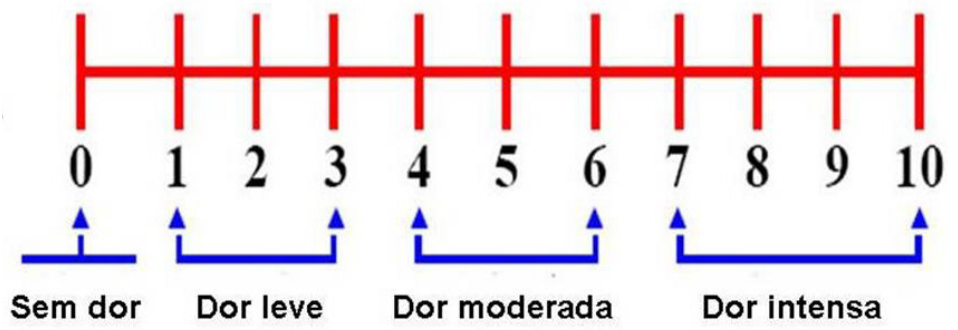

Figura 2. Escala visual/verbal numérica

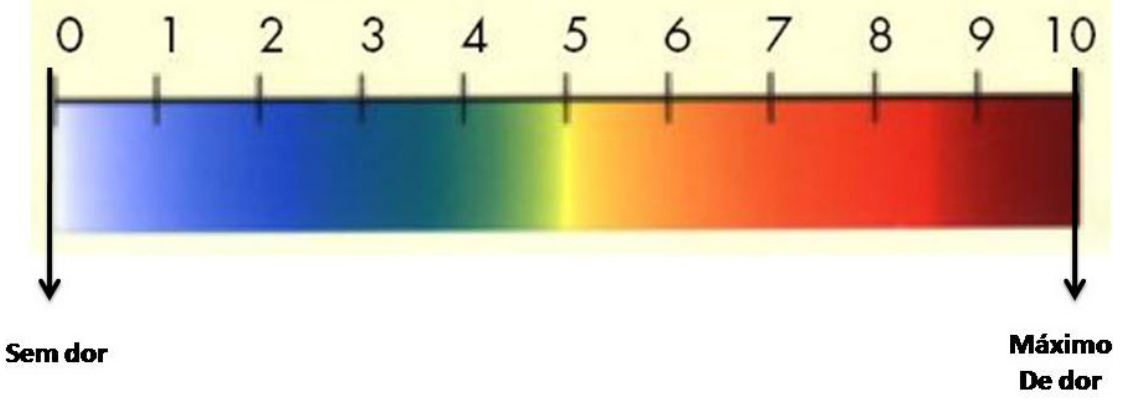

Figura 3. Escala visual analógia

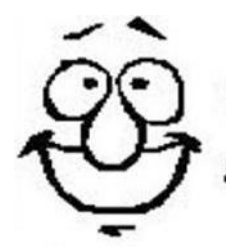

Sem dor

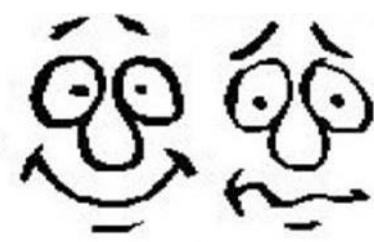

Dor leve

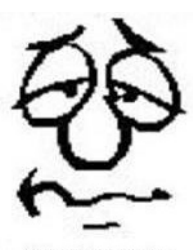

Moderada

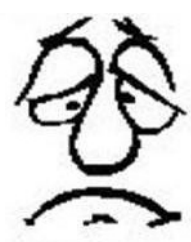

Forte

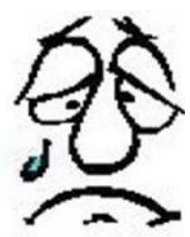

Insuportável

Figura 4. Escala de faces de dor

essa avaliação pelo paciente, ou seja, que o enfermeiro comparasse a face de dor do cliente com as da escala seria inadequado. Sob o contexto do paciente crítico, mesmo com baixo nível de sedação, visando à avaliação holística, apenas um parâmetro de avaliação (face de dor) pode ser insuficiente para a identificação correta de um episódio álgico. O indivíduo em UTI está exposto às diversas questões que podem impossibilitá-lo de manifestar algo que o incomode, que não seja dor propriamente dita, mas devido à incapacidade de usar a fala, vale-se da expressão facial para fazê-lo. Um exemplo disso seria uma UTI com grande quantidade de ruídos (alarmes, vozes altas, etc.) que podem incomodar o paciente e causar um desconforto que não se caracteriza como dor. Neste caso ele pode, por exemplo, "franzir a testa", como indicado na segunda face da EFD, mas não apresentar dor. No entanto, para um paciente hospitalizado em uma enfermaria, clinicamente estável, sem grande quantidade de dispositivos e procedimentos invasivos, esta escala seria facilmente aplicada.

Já o questionário de dor McGill avalia a dor a crônica em adultos através de autorrelato. Também exige que o paciente esteja acordado e orientado. Sua dor é vista através de um caráter individual e subjetivo. O questionário é dividido em 20 categorias, onde cada uma possui diversas opções de resposta. As opções são lidas para o paciente e ele deve escolher, dentro de cada categoria, ao menos uma que represente a dor que ele está sentindo (Figura 5). As categorias de 1 a 10 correspondem às respostas sensitivas, as de 11 a 15 são de caráter afetivo, o 16 é de caráter avaliativo e de 17 a 20 misturam as outras 


\begin{tabular}{|c|c|c|c|c|}
\hline $\begin{array}{l}\text { 1-vibragão } \\
\text { 2-tremor } \\
\text { 3-pulsante } \\
\text { 4- latejante } \\
\text { 5-como batida } \\
\text { 6-como pancada }\end{array}$ & $\begin{array}{l}\text { 1-pontada } \\
\text { 2-choque } \\
3 \text { - tiro }\end{array}$ & $\begin{array}{l}\text { 1-agulhada } \\
\text { 2-perfiurante } \\
\text { 3-facada } \\
\text { 4-punhalada } \\
\text { 5-em langa }\end{array}$ & $\begin{array}{l}\text { 1- fina } \\
\text { 2-cortante } \\
3 \text { - estragalhada }\end{array}$ & $\begin{array}{l}\text { 1-beliscão } \\
2 \text { - aperto } \\
3 \text {-mordida } \\
\text { 4-cólica } \\
5 \text { - esmagamento }\end{array}$ \\
\hline $\begin{array}{l}\text { 1-fisgada } \\
\text { 2-puxão } \\
\text { 3-em torção }\end{array}$ & $\begin{array}{l}\text { 1-calor } \\
\text { 2-queimacão } \\
\text { 3-fervente } \\
\text { 4-embrasa }\end{array}$ & $\begin{array}{l}\text { 8. } \\
\text { 1-formigamento } \\
2 \text {-coceira } \\
\text { 3-ardor } \\
4 \text { - ferroada }\end{array}$ & $\begin{array}{l}\text { 9. } \\
\text { 1-mal localizada } \\
2 \text {-dolorida } \\
3 \text {-machucada } \\
\text { 4-doida } \\
5 \text {-pesada }\end{array}$ & $\begin{array}{l}10 . \\
\text { 1-sensivel } \\
\text { - esticada } \\
\text { - esfolante } \\
\text { - rachando }\end{array}$ \\
\hline $\begin{array}{l}11 . \\
1-\text { cansativa } \\
2 \text { - exaustiva }\end{array}$ & $\begin{array}{l}12 . \\
2 \text { - enjoada }\end{array}$ & \begin{tabular}{|l}
13. \\
1 - amedrontada \\
2- apavorante \\
3- aterrorizante
\end{tabular} & $\begin{array}{l}14 . \\
1 \text { - castigante } \\
2 \text { - atormenta } \\
3 \text { - cruel } \\
\text { 4-maldita } \\
5 \text { - mortal }\end{array}$ & \begin{tabular}{|c|}
15. \\
$1-$ miserável \\
$2-$ enlouquecedora
\end{tabular} \\
\hline $\begin{array}{l}\text { 1-chata } \\
\text { 2-que incomoda } \\
\text { 3-desgastante } \\
\text { 4-forte } \\
\text { 5-insuportável }\end{array}$ & $\begin{array}{l}17 \text { - espalha } \\
2-\text { irradia } \\
\text { 3-penetra } \\
4 \text { - atravessa }\end{array}$ & $\begin{array}{l}18 . \\
1 \text {-aperta } \\
2 \text {-adormece } \\
3 \text {-repura } \\
\text { 4- espreme } \\
5 \text {-rasga }\end{array}$ & $\begin{array}{l}19 . \\
1-\text { fria } \\
2-\text { gelada } \\
3-\text { congelante }\end{array}$ & $\begin{array}{l}20 . \\
1 \text { - aborrecida } \\
\text { 2-dá náuseas } \\
\text { 3-agonizante } \\
\text { 4-pavorosa } \\
\text { 5-torturante }\end{array}$ \\
\hline
\end{tabular}

Figura 5. Questionário de dor McGill

categorias. Após a aplicação do questionário o profissional precisa somar quantas palavras o paciente citou para descrever sua dor (mínimo de 20). Além disso, ainda é preciso somar a intensidade da dor descrita pelo paciente. Ele precisa indicar, numa escala de zero a cinco, o nível de intensidade de cada palavra que o mesmo escolheu em cada categoria. Este método ainda pode ser associado a um diagrama corporal para que o paciente indique, no desenho, o local da sua dor. Sua aplicação pode levar de três a cinco minutos aproximadamente, variando conforme o padrão de resposta do indivíduo. Exige maior treinamento por parte do profissional para interpretar os resultados da escala e escolher a melhor maneira de intervir. ${ }^{10,11}$

Para a aplicação desta escala o paciente precisar ter uma alta capacidade cognitiva, pois além de entender o princípio da mesma deve ser capaz de comparar sua dor com as palavras ditas e escolher somente uma opção dentro de cada categoria. Desta forma, entende-se que não é válida em situações de dor extrema, pois o indivíduo deve ter tempo e clareza para pensar e responder o que mais se assemelha ao que está sentindo. Isso dificulta que esta escala seja aplicada a pacientes em terapia intensiva, excluindo-se aqueles estáveis clinicamente com as capacidades supracitadas para responder ao questionário. Em geral, os pacientes críticos não estarão aptos para serem avaliados desta maneira, devido à sedação e/ou à dificuldade de se expressar com palavras.

As dificuldades na fala diminuem a troca de informações entre o paciente e o profissional, prejudicando o vínculo afetivo, consequentemente diminuindo a segurança, pois a falta de comunicação pode provocar frustração, medo e isolamento. ${ }^{2}$

Para pacientes com limitação cognitiva grave, existem critérios que podem sugerir episódios de dor, como: câncer metastático, doenças articulares com deformidades, úlceras de decúbito, pós-operatório de cirurgias de médio e grande porte, queimados, exames e procedimentos invasivos, procedimentos potencialmente dolorosos (banho, troca de curativos e fisioterapia) e presença de drenos. Ainda de acordo com esta pesquisa, diante de tais situações, o paciente pode apresentar os seguintes comportamentos: adoção de postura de proteção, movimento de retirada ao estímulo doloroso, agitação resistente mesmo após medidas farmacológicas de conforto, diminuição do 
nível de atividade, expressão facial de sofrimento, vocalização (gemência e choro), alteração do padrão do sono e diminuição do apetite ou ingesta alimentar. ${ }^{8}$ No entanto, deve-se ressaltar que estes comportamentos são apenas sugestivos de dor, não podendo ser usados isoladamente para a confirmação da mesma.

A escala de dor comportamental (Behavioral Pain Scale - BPS) (Figura 6) é fácil de ser utilizada e é aplicável para avaliar a dor de pacientes sedados e/ou em ventilação mecânica. Seu escore vai de 3 a 12 através da observação da expressão facial, de movimentos do corpo, de tensão muscular e sincronia com o ventilador. Se o escore for maior que 6 é considerado

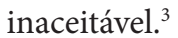

A expressão facial é o item que mais contribui para a avaliação da dor, seguida de movimentos dos membros e da aceitação da ventilação. São comportamentos de dor: careta, testa franzida, rigidez, retração, pálpebras cerradas apertadas, nariz franzido, lábio superior levantado, verbalização e punhos cerrados. A aceitação da ventilação mecânica pode ser afetada por hipoxemia, broncoespasmo e secreção. ${ }^{3}$

Esta escala contempla a maior quantidade de parâmetros a serem observados no paciente crítico, visando avaliá-lo de forma completa.

\begin{tabular}{|l|l|}
\hline Expressīo facial & \\
Relaxada & 1 \\
Totalmente tensa & 2 \\
Fazendo careta & 3 \\
& 4 \\
\hline Movimentos dos membros superiores & \\
Relaxado & 1 \\
Parcialmente flexionado & 2 \\
Totalmente flexionado & 3 \\
Totalmentecontraido & 4 \\
\hline Ventilacia Mecinnica & \\
Tolerando movimentos & \\
Tossindo, mas tolerandoa maior partedo tempo & 2 \\
Latandocontrao ventilador & 3 \\
Impossibilidadedecontroledo ventilador & 4 \\
\hline
\end{tabular}

Figura 6. Escala de dor comportamental
É importante que ao ser aplicada apresente-se estratificada para que seja possível visualizar qual a pontuação obtida em cada critério. Mesmo que não seja possível disponibilizar a escala completa no mesmo impresso utilizado para registrar os sinais vitais, para que o profissional a preencha, faz-se necessário que estas anotações indiquem o somatório realizado para se alcançar o resultado final. Por exemplo: BPS $(3+3+2=$ 8). Neste exemplo é possível identificar o valor obtido em cada categoria.

A escala de dor ideal deve ser de fácil aplicação e interpretação, possibilitar que as pequenas alterações já sejam avaliadas, examinar a sedação a fim de realizar a correta titulação de fármacos, além de observar a agitação do paciente. $^{3}$

O enfermeiro é o profissional de referência da equipe assistencial, por isso cabe a ele garantir que as ações relacionadas ao gerenciamento da dor sejam aplicadas e que o paciente esteja devidamente assistido. Desta forma, de posse da escala de dor e da prescrição médica, que indica os medicamentos que devem ser administrados para controle da dor caso necessário, assim como seus intervalos pré-estabelecidos, o enfermeiro terá ferramentas para agir no controle eficaz da dor do paciente crítico. Este profissional poderá de igual forma executar ações não farmacológicas para o controle da dor, como melhor posicionamento no leito, uso de compressas, massagem de conforto ou erradicação do agente causador da dor, se possível. ${ }^{8}$

Uma ação importante no controle da dor é a reavaliação periódica através da mesma escala usada inicialmente, ou seja, a partir do momento que se escolheu a escala que melhor se adeque ao paciente, a mesma deve ser utilizada nas reavaliações. Caso haja melhora ou piora no quadro e seja preciso mudar a escala usada, é importante que seja relatado no prontuário do paciente para que os profissionais que realizarão as avaliações posteriores estejam informados sobre o método que está sendo empregado.

O profissional deve atentar para a indicação de cada escala e se a idade do paciente o permite 
se submeter a essa avaliação. Existem escalas aplicáveis somente a crianças, outras a adultos e ainda aquelas que se aplicam a ambos, ou seja, existem diversos fatores a serem observados e avaliados antes de proceder a mensuração da dor. $^{8}$

Os pacientes em situação de dor aguda ou crônica persistente devem ser reavaliados, no máximo, a cada uma hora após intervenção terapêutica. ${ }^{8}$ Já os outros indivíduos podem ser avaliados, quanto à dor, de acordo com a rotina institucional de aferição dos outros sinais vitais. ${ }^{7}$

Cabe apontar que, para que o cuidado seja integral e individualizado é preciso que tanto a escolha da escala quanto as avaliações e as intervenções sejam registradas, pelo profissional de enfermagem que realizou tais etapas, de forma correta e precisa.

\section{Conclusão}

As escalas de dor devem ser consideradas pela equipe de enfermagem como valiosos instrumentos para o manejo correto da dor dos pacientes, principalmente daqueles internados em UTIs.

Procurar conhecer e ter uma percepção crítica e reflexiva dos modelos de escalas existentes na literatura, através das revisões bibliográficas, é de suma importância para a capacitação e o aperfeiçoamento profissional.

A enfermagem precisa realizar continuamente análises das escalas disponíveis a fim de que se escolha aquela mais direcionada para o tipo de paciente em questão. Esta conduta não deve ser praticada de forma padronizada e sim conforme a demanda do paciente.

Estudos como este são importantes porque visam estimular a construção de produção científica direcionada à temática da dor. A enfermagem precisa se empoderar da sua capacidade de gerar este tipo de conhecimento, visto que é a classe que atua mais tempo diretamente com os pacientes propensos a terem dificuldades em se expressar nos episódios álgicos, sobretudo os considerados críticos

\section{Referências}

1. Ciena AP, Gatto R, Pacini VC, Picanço VV, Magno IMN, Loth EA. Influência da intensidade da dor sobre as respostas nas escalas unidimensionais de mensuração da dor em uma população de idosos e de adultos jovens. Semina: Ciências Biológicas e da Saúde. 2008;29(2):201-12.

2. Barbosa TP, Beccaria LM, Pereira RAM. Avaliação da experiência de dor pósoperatória em pacientes de unidade de terapia intensiva. Rev Bras Ter Intensiva. 2011;23(4):470-7.

3. Sakata RK. Analgesia e Sedação em Unidade de Terapia Intensiva. Rev Bras Anestesiol. 2010;60(6):648-58.

4. Magalhães PAP, Mota FA, Saleh CMR, Secco LMD, Fusco SRG, Gouvêa AL. Percepção dos profissionais de enfermagem frente à identificação, quantificação e tratamento da dor em pacientes de uma unidade de terapia intensiva de trauma. Revista Dor. 2011;12(3):221-5.

5. Pompeo DA, Rossi LA, Galvão CM. Revisão integrativa: etapa inicial do processo de validação de diagnóstico de enfermagem. Acta paulista de enfermagem. 2009;22(4):4348.

6. Mendes KDS, Silveira RCCP, Galvão CM. Revisão integrativa: método de pesquisa para a incorporação de evidências na saúde e na enfermagem. Texto \& contexto enfermagem. 2008;17(4):758-64.

7. Saça CS, Carmo FA, Arbuleia JPS, Souza RCX, Alves SA, Rosa BA. A dor como 5o sinal vital: atuação da equipe de enfermagem no hospital privado com gestão do Sistema Único de Saúde. J Health Sci Inst. 2010;28(1):35-41.

8. Hospital Israelita Albert Einstein. Gerenciamento da dor na SBIBHAE. 2010.

9. Andrade FA, Pereira LV, Sousa FAEF. Mensuração da dor no idoso: uma revisão. Rev Lat Am Enfermagem. 2006;14(2):271-6.

10. Bottega FH, Fontana RT. A dor como quinto sinal vital: utilização da escala de avaliação por enfermeiros de um hospital geral. Texto \& contexto enfermagem. 2010;19(2):283-90.

11. Morete MC, Minson FP. Instrumentos para a avaliação da dor em pacientes oncológicos. Revista Dor 2010;11(1):74-80. 